\title{
Soil Pesticide Residues in Orchard based Land Use Systems across Different Agro-climatic Zones of Himachal Pradesh
}

\author{
Sukh Pal Singh Brar* and I.D. Sharma
}

Department of Entomology and Apiculture, Dr. Y.S. Parmar University of Horticulture and Forestry, Nauni, Solan - 173230 Himachal Pradesh, India

*Corresponding author

\section{A B S T R A C T}

\section{Keywords}

Pesticide residue, $\mathrm{HCH}, \mathrm{DDT}$, Chlorpyrifos, Endosulfan, Orchards

Article Info

Accepted:

10 March 2019

Available Online:

10 April 2019
The soils were collected from different orchard and uncultivated fields, from four agroclimatic zones of Himachal Pradesh viz., 1) sub tropical sub montane and low hills 2) temperate sub-humid mid-hills 3) wet temperate and high hills 4) dry temperate high hills cold desert, and pesticide residue study was carried out in Toxicology Laboratory, Department of Entomology and Apiculture, Dr. Y.S. Parmar University of Horticulture and Forestry, Nauni, Solan, Himachal Pradesh, India. More than 90 per cent soil samples analysed from different zones of the state have shown presence of various pesticide residues viz., DDT, HCH, endosulfan, chlorpyrifos, pyrethroids, dicofol and chlorothalonil. Apple orchard soils were found to contain highest ensdosulfan residues followed by $\mathrm{HCH}$ and DDT residues. In mango orchard soils dicofol was detected in addition to the apple soil contaminants. Further, the most common insecticide residues in Himachal soils are DDT, HCH, endosulfan, chlorpyrifos, dicofol, pyrethroids and chlorothalonil in the order of decreasing contamination.

\section{Introduction}

The state of the Himachal Pradesh specialises in the genre of horticulture. Fruits grow in HP covering an absolute area of 2.07 Lac hectares. Himachal Pradesh has made a tremendous progress in production of fruits during the last two decades. The total production of fruits in the state is not less than 5.00 Lac MTs. Nonetheless there is a speedy progress in biological control measures, yet pesticidal application cannot be dispensed and still it remains as one of the major weapons in the hands of farmers to control pests. Due to market driven demand, to increase the intensity of production customarily farmers have been using agrochemicals with a high dosage to meet this demand and enhance income through increased production. The situation seems to be goaded in the years to come due to ever increasing demographic growth and dearth of cultivable land. These pesticides can enter ground water resources and surface run-off during rainfall, thus causative of environmental contamination. Because of their widespread use, these are 
detected soil, water and air (Murugan et al., 2013; Sharma et al., 2015; Bakshi, 2016). Thus, along with development of HVC crops, many second-generation issues are emerging.

At present, out of the 145 pesticides registered in India about 40 are in use on various crops in the state and this consumption is increasing at alarming rate. In India and elsewhere in the world, synthetic pesticides have been very popular for their use by farmers because of their broad spectrum of activity, ease in storage, application, and high economic returns. Even though the consumption of pesticides in India is about $400 \mathrm{~g} / \mathrm{ha}$ which is very low as compared to Europe $2 \mathrm{~kg} / \mathrm{ha}$ and $10 \mathrm{~kg} / \mathrm{ha}$ in Japan, yet there is a wide spread contamination of our feed and food commodities and environment with pesticides. Pesticides like DDT, $\mathrm{HCH}, \mathrm{HCB}$, dieldrin and endrin have a long history of use in the world for control of agricultural pests and are typical persistent organic pollutants (Zhang et al., 2006; Wang et al., 2007; Sharma et al., 2015). These are still routinely found in soil, water, air and even in the food chain (Gong et al., 2004; Barriada-Pereira et al., 2005; Concha-Grana et al., 2006). A number of reports have indicated the presence of different groups of pesticide residues in soils from several parts of India (Kumari et al., 2004; Jayashree and Vasudevan, 2006; Bishnu et al., 2008; Sharma et al., 2015) and the world (Manirakiza et al., 2003; Kannan et al., 2003; Dem et al., 2007). In a recent survey, it was found that the food commodities are not only contaminated with pesticide residues but these have also been detected in underground water and in all the major rivers of India which is quite alarming (Agnihotri 1999; Banshtu, 2015; Brar and Sharma, 2016). After (pesticides) application either as foliar spray or soil treatment, its major portion is retained on the surface of soil and remaining will be moved down and ultimately find its way into the aquatic system
(Jain and Agnihotri ,1986). Therefore, to know the status of pesticide residues in orchard land use soils, and investigations were carried out for monitoring these residues in different agroclimatic zones of Himachal Pradesh.

\section{Materials and Methods}

The monitoring of pesticide residues in soils collected from fields with intensive crop production and without cultivation (uncultivated), selected for sampling from four agro-climatic zones of Himachal Pradesh viz., 1) sub tropical sub montane and low hills 2) temperate sub-humid mid-hills 3) wet temperate and high hills and 4) dry temperate high hills cold desert were carried out in Toxicology Laboratory, Department of Entomology and Apiculture, Dr. Y.S. Parmar University of Horticulture and Forestry, Nauni, Solan, Himachal Pradesh, India. Composite soil samples were drawn from each zone having two identified locations and four land use patterns at two times i.e. before flowering/ before harvest and at harvest of crop by using $\mathrm{X}$ and $\mathrm{N}$ system of sampling at 0-15 cm depth. The physico-chemical properties of soil in different zones are given in table 1.

\section{Residue analysis}

Soil containing pesticide residues absorbed on florisil, eluted with hexane and acetone (9:1), clean up on silicagel with $15 \mathrm{ml}$ mixture of hexane and acetone in 9:1 ratio (v/v) was quantified on Gas chromatograph. The soil sample $(1 \mathrm{~kg})$ brought from fields were air dried, mixed thoroughly and sieved through $20 \mathrm{~mm}$ mesh sieve. From each sieved sample two-sub samples of $15 \mathrm{~g}$ each were drawn for further use. These sub samples were analyzed for organochlorines, organophosphates, pyrethroids, cyclodienes and some fungicides (mancozeb and carbendazim). From the processed soils samples, 3-5 sub samples of 
$15 \mathrm{~g}$ each were taken for spiking and technique standardization. Soil samples of $15 \mathrm{~g}$ each were fortified at $1 \mathrm{ppm}$. These samples were processed and peak areas were used to calibrate the integrator using blank for listing quantities of pesticides used in sample. At each level recovery was calculated as follow,

$$
\text { Recovery }(\%)=\frac{\text { Amount added }}{\begin{array}{l}
\text { Amount } \\
\text { recovered }
\end{array}} \times 100
$$

Homogenized soil sample of $15 \mathrm{~g}$ was blended with $0.3 \mathrm{~g}$ florisil and $0.3 \mathrm{~g}$ charcoal in mortar until free flowing. The free flowing soil, florisil and charcoal mixture were placed into sintered column, having $1 \mathrm{~g}$ anhydrous sodium sulphate at its base. After slight tapping, the packed material was eluted with $100 \mathrm{ml}$ of hexane and acetone, (9:1). Eluent was evaporated to dryness in rotary evaporator at $40-50^{\circ} \mathrm{C}$. Residues were redissolved in $5 \mathrm{ml} \mathrm{n}$-hexane for clean up.

The n-hexane containing pesticide residues and plant material was loaded on $1 \mathrm{~g}$ activated silica gel. The column was eluted with $15 \mathrm{ml}$ of 10 per cent acetone in hexane. The eluent was evaporated in rotary evaporator to dryness. Residues were re-dissolved in $5 \mathrm{ml} \mathrm{n}$ hexane and $1 \mu \mathrm{l}$ of it was injected into G.C.

Pesticide residues were detected using Gas Chromatography (Hewlett Packard 5890 Series II with Agilent 3396 III Integrator) with Ultra Performance Capillary column Cross-linked Methyl Silicon Film thickness: 0.33 microns, Int. diameter: $0.20 \mathrm{~mm}$, length: 25 metre $160^{\circ} \mathrm{C}$ for 2 minutes, final temp. $260^{\circ} \mathrm{C} @ 3.5^{\circ} \mathrm{C} / \mathrm{min}$; temperature ECD: $300^{\circ} \mathrm{C}$, NPD: $270^{\circ} \mathrm{C}$; detector temperature was $260^{\circ} \mathrm{C}$; Injection temp. gas flow- Iolar Nitrogen @ $4 \mathrm{ml} / \mathrm{min} .$, septa purge @ 2 $\mathrm{ml} / \mathrm{min}$., make up gas $25 \mathrm{ml} / \mathrm{min}$, Hydrogen 1 $\mathrm{ml} / \mathrm{min}$. The residues estimation methods (Table 2) followed for different pesticides in the present study are given in table 2. The residues of dithiocarbamates were estimated as per the method of Dubey and Stan (1998). Dithiocarbamate residues were estimated according to method described by Dubey et al., (1999) on $\mathrm{CS}_{2}$ basis.

\section{Results and Discussion}

\section{Pesticide residues in apple orchard soils}

Pesticide residues were monitored in apple orchard soils collected from four locations viz., Kukumseri, Rekong Peo, Bajaura and Mashobra at two times i.e. before flowering and at harvest. The data presented in Table 3 reveal that in Kukumseri samples $\mathrm{HCH}$ isomers viz., $\alpha-\mathrm{HCH}(0.002 \mathrm{mg} / \mathrm{kg}), \beta-\mathrm{HCH}$ $(0.004 \mathrm{mg} / \mathrm{kg})$ and $\gamma-\mathrm{HCH}(0.016 \mathrm{mg} / \mathrm{kg})$ were detected before flowering whereas, only endosulfan $(0.019 \mathrm{mg} / \mathrm{kg})$ was found at harvest stage.

At Rekong Peo, only chlorpyrifos (0.004 $\mathrm{mg} / \mathrm{kg}$ ) was detected before flowering, whereas, $\alpha-\mathrm{HCH} \quad(0.002 \mathrm{mg} / \mathrm{kg}), \quad \gamma-\mathrm{HCH}$ $(0.004 \mathrm{mg} / \mathrm{kg}), \mathrm{p}, \mathrm{p}$-DDE $(0.006 \mathrm{mg} / \mathrm{kg}), \beta$ endosulfan $(0.008 \mathrm{mg} / \mathrm{kg})$ and endosulfansulphate $(0.311 \mathrm{mg} / \mathrm{kg})$ were detected at harvest time. The samples collected from Bajaura location contained no residue at flowering stage. However, at harvest stage the concentration of $p, p$ '-DDE, $\beta$-endosulfan, p,p'-DDT, chlorpyrifos-methyl, and chlorpyrifos were detected as 0.002, 0.006, $0.003,0.006$ and $0.004 \mathrm{mg} / \mathrm{kg}$, respectively.

At Mashobra, none of the soil samples were found contaminated with any pesticide before flowering, whereas, at harvest stage the residues of pesticides viz., $\alpha-\mathrm{HCH}, \beta-\mathrm{HCH}, \gamma-$ $\mathrm{HCH}, \alpha$-endosulfan, $\mathrm{p}, \mathrm{p}$ '-DDE, $\beta$-endosulfan, endosulfan-sulphate, p,p'-DDT, chlorpyrifos and fenvalerate were detected as 0.004, 0.002, $0.003,0.002,0.001,0.012,0.023,0.003$, 0.005 and $0.011 \mathrm{mg} / \mathrm{kg}$, respectively. 
Pesticide residues in mango, plum and kinnow orchard soils of Himachal Pradesh

The pesticide residues at two sampling times i.e. before flowering and at harvest revealed that only $\beta$-endosulfan $(0.002 \mathrm{mg} / \mathrm{kg})$ was detected in mango orchard soil before flowering (Table 4). At harvest, however, $\alpha$ $\mathrm{HCH}(0.001 \mathrm{mg} / \mathrm{kg}), \gamma-\mathrm{HCH}(0.003 \mathrm{mg} / \mathrm{kg})$, $\delta$ - $\mathrm{HCH}(0.053 \mathrm{mg} / \mathrm{kg})$, dicofol $(0.021 \mathrm{mg} / \mathrm{kg})$, o,p'-DDE $\quad(0.013 \quad \mathrm{mg} / \mathrm{kg}), \quad \alpha$-endosulfan $(0.037 \mathrm{mg} / \mathrm{kg}), \mathrm{p}, \mathrm{p}$ '-DDE $(0.007 \mathrm{mg} / \mathrm{kg}), \beta$ endosulfan $(0.011 \mathrm{mg} / \mathrm{kg}), \mathrm{p}, \mathrm{p}$-DDD $(0.003$ $\mathrm{mg} / \mathrm{kg})$, o,p'-DDT (0.003 mg/kg), endosulfansulphate $(0.030 \mathrm{mg} / \mathrm{kg})$ and $\mathrm{p}, \mathrm{p}$-DDT $(0.009$ $\mathrm{mg} / \mathrm{kg}$ ) were found.

The soils of plum orchards of Solan area, contained residues of $\gamma-\mathrm{HCH}(0.003 \mathrm{mg} / \mathrm{kg})$ and p,p'-DDE $(0.033 \mathrm{mg} / \mathrm{kg})$ before flowering and p,p'-DDE $(0.006 \mathrm{mg} / \mathrm{kg}), \mathrm{p}, \mathrm{p}^{\prime}-\mathrm{DDT}$ $(0.003 \mathrm{mg} / \mathrm{kg})$ and chlorpyrifos (0.004 $\mathrm{mg} / \mathrm{kg}$ ), at harvest stage. The kinnow orchard soils of Dhaulakuan area were found contaminated with chlorpyrifos-methyl (0.006 and $0.004 \mathrm{mg} / \mathrm{kg}$ ), at both the stages of sampling. At Jachh, the residues of $\beta-\mathrm{HCH}$ $(0.003 \mathrm{mg} / \mathrm{kg})$ and $\beta$-endosulfan $(0.006$ $\mathrm{mg} / \mathrm{kg}$ ) were found before flowering and no residues of other pesticides were detected at harvest. The experiment conducted for monitoring the pesticides residues in orchard soils (apple, plum, mango and kinnow) indicated that the apple and mango soils were contaminated with DDT, $\mathrm{HCH}$ and endosulfan residues (Table 5). Apple orchard soils were found to contain highest endosulfan $(0.006-0.319 \mathrm{mg} / \mathrm{kg})$ followed by $\mathrm{HCH} \quad(<0.002-0.022 \mathrm{mg} / \mathrm{kg})$ and DDT $(<0.001-0.006 \mathrm{mg} / \mathrm{kg})$ residues in four different locations (Kukumseri, Rekong Peo, Bajaura and Mashobra) of Himachal Pradesh. Like apple soils, mango orchard soils also accumulated the highest mean contents of $\Sigma$ endosulfan $(0.073 \mathrm{mg} / \mathrm{kg})$ followed by $\Sigma$ $\mathrm{HCH}(0.057 \mathrm{mg} / \mathrm{kg}), \Sigma$-DDT $(0.034 \mathrm{mg} / \mathrm{kg})$ and dicofol $(0.021 \mathrm{mg} / \mathrm{kg})$. The occurrence of high level of endosulfan residues in apple and mango orchard soils has reflected the use of this insecticide in regular interval on the crops for the control of pests. The plum soils contained more $\Sigma$-DDT $(0.022 \mathrm{mg} / \mathrm{kg})$ than $\gamma$ $\mathrm{HCH}(0.003 \mathrm{mg} / \mathrm{kg})$ residues and only lower residue levels of $\beta-\mathrm{HCH}(0.003 \mathrm{mg} / \mathrm{kg})$ and $\beta$ endosulfan $(0.006 \mathrm{mg} / \mathrm{kg})$ were found in kinnow soils. The present findings are in conformity with the findings of Harris and Sans $(1969,1971)$ who reported that apple orchard soils had the highest organochlorine residues in the cropping practices. They reported both DDT and dicofol in orchard soils. The higher residues of organochlorines as compared to present findings have been reported by Frank et al., (1976). According to them the most frequently found insecticides were DDT, TDE and their metabolites DDE which were not longer in use. Apple and peach orchards had the highest mean residues of 43.3 and $9.22 \mathrm{ppm}$ of $\Sigma$-DDT, respectively. Other organochlorine insecticides found were endosulfan, endrin and methoxychlor residues were below $1 \mathrm{ppm}$ with the exception of endosulfan. The lower residue levels may be due to the complete restriction on their use in public health and on fruit crops. Different orchards were studied by Harris and Sans (1969, 1971), who also suggested a continuing decline in $\Sigma$-DDT residues. The results also showed that unlike organochlorine pesticides, the presence of organophosphorus and pyrethriod compounds were present least in apple, mango, plum and kinnow soils. Among organophosphorus insecticides, only chlorpyrifos was detected in apple, plum and kinnow orchards with highest mean residues of $0.010, \quad 0.004$ and $0.005 \mathrm{mg} / \mathrm{kg} \quad \Sigma$ chlorpyrifos, respectively. Though number of pyrethroids have been recommended for the control of fruit pests, yet only the fenvalerate $(0.011 \mathrm{mg} / \mathrm{kg})$ and that too in apple sample of Mashobra was detected (Table 5). 


\section{Pesticide residues in uncultivated fields}

Pesticide residues were also monitored in uncultivated field soils from eight locations viz., Kukumseri, Rekong Peo, Bajaura, Mashobra, Bilaspur, Solan, Dhaulakuan and Jachh.

The data presented in Table 6 show that in Kukumseri samples, $\alpha-\mathrm{HCH}(0.002 \mathrm{mg} / \mathrm{kg})$, $\beta-\mathrm{HCH}(0.009 \mathrm{mg} / \mathrm{kg}), \gamma-\mathrm{HCH}(0.003 \mathrm{mg} / \mathrm{kg})$, dicofol $(0.025 \mathrm{mg} / \mathrm{kg})$, $\alpha$-endosulfan $(0.005$ $\mathrm{mg} / \mathrm{kg})$ and p,p'-DDE $(0.003 \mathrm{mg} / \mathrm{kg})$ were found. At Rekong Peo location, the residues of $\gamma-\mathrm{HCH} \quad(0.002 \mathrm{mg} / \mathrm{kg}), \quad \delta-\mathrm{HCH} \quad(0.002$ $\mathrm{mg} / \mathrm{kg})$, dicofol $(0.006 \mathrm{mg} / \mathrm{kg})$, o,p'-DDE $(0.004 \mathrm{mg} / \mathrm{kg}), \alpha$-endosulfan $(0.004 \mathrm{mg} / \mathrm{kg})$,
p,p'-DDE ( $0.002 \mathrm{mg} / \mathrm{kg}), \beta$-endosulfan (0.016 $\mathrm{mg} / \mathrm{kg})$ and endosulfan-sulphate $(0.029$ $\mathrm{mg} / \mathrm{kg}$ ) were detected. In the samples collected from Bajaura location, the residues of dicofol $(0.004 \mathrm{mg} / \mathrm{kg}), \alpha$-endosulfan $(0.003$ $\mathrm{mg} / \mathrm{kg}), \quad \mathrm{p}, \mathrm{p}^{\prime}-\mathrm{DDE} \quad(0.049 \quad \mathrm{mg} / \mathrm{kg}), \quad \beta-$ endosulfan $(0.002 \mathrm{mg} / \mathrm{kg})$, p,p'-DDD (0.070 $\mathrm{mg} / \mathrm{kg})$ and o,p'-DDT $(0.015 \mathrm{mg} / \mathrm{kg})$ were obtained whereas, in samples collected from Mashobra the residues of p,p'-DDD (0.196 $\mathrm{mg} / \mathrm{kg})$ and $\alpha$-methrin $(0.026 \mathrm{mg} / \mathrm{kg})$ were recorded and in Bilaspur area, the residues of $\alpha-\mathrm{HCH}, \quad \gamma-\mathrm{HCH}$, dicofol, p,p'-DDE, p,p'DDD, o,p'-DDT and p,p'-DDT were detected at concentrations of $0.003,0.013,0.003$, $0.126, \quad 0.035, \quad 0.015$ and $0.153 \mathrm{mg} / \mathrm{kg}$, respectively.

Table.1 Physico-chemical properties of soils collected from Himachal Pradesh

\begin{tabular}{|l|c|c|}
\hline Zone & $\mathbf{p H}$ & Per cent organic matter \\
\hline $\begin{array}{l}\text { Dry temperate, high hills, cold } \\
\text { desert (Zone 4) }\end{array}$ & 7.12 & 8.24 \\
\hline & 6.88 & 5.84 \\
\hline $\begin{array}{l}\text { Wet temperate and high hills } \\
\text { (Zone 3) }\end{array}$ & 6.50 & 7.34 \\
\hline & 6.15 & 10.34 \\
\hline $\begin{array}{l}\text { Temperate sub-humid mid-hills } \\
\text { (Zone 2) }\end{array}$ & 7.00 & 10.39 \\
\hline & 6.82 & 4.13 \\
\hline & 6.80 & 8.17 \\
\hline $\begin{array}{l}\text { Sub-tropical sub-mountane and } \\
\text { low hills (Zone 1) }\end{array}$ & 7.22 & 8.53 \\
\hline & 6.66 & 8.99 \\
\hline
\end{tabular}


Table. 2 Various residues estimation methods

\begin{tabular}{|c|c|c|}
\hline Sr. No. & Name of pesticide & Residues estimation method \\
\hline 1. & DDT & Colorimetric \\
\hline 2. & Parathion & Colorimetric \\
\hline 3. & Benezene hexachloride & Colorimetric \\
\hline 4. & Aldrin & Colorimetric \\
\hline 5. & Dieldrin & Colorimetric \\
\hline 6. & Dieldrin & GC-ECD \\
\hline 7. & Disyston & Colorimetric \\
\hline 8. & $\gamma \mathrm{HCH}$ (Hexachlorocyclohexane & TLC/GC \\
\hline 9. & Carbaryl & Spectrophotometer \\
\hline 10. & Dimethoate and thimet & GC emission spectroscopic \\
\hline 11. & Carbofuran & GC-NPD \\
\hline 12. & Disulfoton & GLC \\
\hline 13. & Carbofuran & Colorimetric \\
\hline 14. & DDT & TLC \\
\hline 15. & Organophosphorus & Colorimetric \\
\hline 16. & $\begin{array}{l}\text { Benomyl and Methyl N ( } 2 . \\
\text { Benzimidazole) carbamate }\end{array}$ & GC-ECD \\
\hline 17. & OP & TLC \\
\hline 18. & Chlorophenol & GC \\
\hline 19. & Propyzamide & GC-ECD \\
\hline 20. & Metribuzin & $\begin{array}{l}\text { GC-N-specific Alkali flame ionization } \\
\text { detector }\end{array}$ \\
\hline 21. & $\begin{array}{l}\text { Quinalphos, disulfoton, } \\
\text { monocrotophos }\end{array}$ & TLC \\
\hline 22. & $\begin{array}{l}\text { Benomyl and Methyl N (2. } \\
\text { Benzimidazole) carbamate }\end{array}$ & Voltametric determination \\
\hline 23. & Fluzifop-butyl & GLC-N-specific detector \\
\hline 24. & Dichlobenil & HPLC \\
\hline 25. & Pyrethroids & GC \\
\hline 26. & Methyl-parathion & HPLC \\
\hline 27. & Butachlor & GC-ECD \\
\hline 28. & DDT & ELISA \\
\hline 29. & Atrazine & ELISA \\
\hline 30. & Triazine and chloroaectanilide & GC-NPD or GC-MS \\
\hline
\end{tabular}


Table.3 Pesticide residues in apple orchard soils of Himachal Pradesh

\begin{tabular}{|c|c|c|c|c|c|c|c|c|c|c|c|c|}
\hline \multirow[t]{2}{*}{ Location } & \multirow{2}{*}{$\begin{array}{l}\text { Sampling } \\
\text { time }\end{array}$} & \multicolumn{11}{|c|}{ Residues (mg/kg) } \\
\hline & & $\begin{array}{c}\alpha- \\
\mathrm{HCH}\end{array}$ & $\begin{array}{c}\beta- \\
\mathrm{HCH}\end{array}$ & $\begin{array}{c}\gamma- \\
\mathrm{HCH}\end{array}$ & $\begin{array}{c}\alpha- \\
\text { endosulfan }\end{array}$ & $\begin{array}{l}\mathrm{p}, \mathrm{p}{ }^{\prime}- \\
\text { DDE }\end{array}$ & $\begin{array}{c}\beta- \\
\text { endosulfan }\end{array}$ & $\begin{array}{c}\text { endosulfan } \\
\text { sulphate }\end{array}$ & $\begin{array}{l}\mathrm{p}, \mathrm{p}^{\prime}- \\
\text { DDT }\end{array}$ & $\begin{array}{c}\text { chlorpyrifos- } \\
\text { methyl }\end{array}$ & chlorpyrifos. & fenvalerate \\
\hline \multirow[t]{3}{*}{ Kukumseri } & $\begin{array}{l}\text { Before } \\
\text { flowering }\end{array}$ & 0.002 & 0.004 & 0.016 & ND & ND & ND & ND & ND & ND & ND & ND \\
\hline & At harvest & ND & ND & ND & ND & ND & 0.019 & ND & ND & ND & ND & ND \\
\hline & & & & & & & & & & & & ND \\
\hline \multirow[t]{2}{*}{ Rekongpeo } & $\begin{array}{l}\text { Before } \\
\text { flowering }\end{array}$ & ND & ND & ND & ND & ND & ND & ND & ND & ND & 0.004 & \\
\hline & At harvest & 0.002 & ND & 0.004 & ND & 0.006 & 0.008 & 0.311 & ND & ND & ND & ND \\
\hline \multirow[t]{2}{*}{ Bajaura } & $\begin{array}{l}\text { Before } \\
\text { flowering }\end{array}$ & ND & ND & ND & ND & ND & ND & ND & ND & ND & ND & ND \\
\hline & At harvest & ND & ND & ND & ND & 0.002 & 0.006 & ND & 0.003 & 0.006 & 0.004 & ND \\
\hline \multirow[t]{2}{*}{ Mashobra } & $\begin{array}{l}\text { Before } \\
\text { flowering }\end{array}$ & ND & ND & ND & ND & ND & ND & ND & ND & ND & ND & ND \\
\hline & At harvest & 0.004 & 0.002 & 0.003 & 0.002 & 0.001 & 0.012 & 0.023 & 0.003 & ND & 0.005 & 0.011 \\
\hline
\end{tabular}


Table.4 Pesticide residues in mango, plum and kinnow orchard soils of Himachal Pradesh

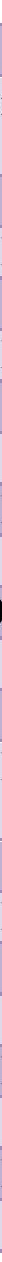


Table.5 Average residues of various pesticides in orchard soils

\begin{tabular}{|c|c|c|c|c|c|c|}
\hline Crop & $\Sigma \mathrm{HCH}$ & $\sum$ DDT & $\begin{array}{c}\text { Dicof } \\
\text { ol }\end{array}$ & $\begin{array}{c}\sum \text { Endosulf } \\
\text { an }\end{array}$ & $\begin{array}{l}\sum \text { Chlorpyri } \\
\text { fos }\end{array}$ & $\begin{array}{c}\sum \text { Pyretho } \\
\text { ids }\end{array}$ \\
\hline \multicolumn{7}{|c|}{ ( } \\
\hline $\begin{array}{l}\text { i) } \\
\text { Kukumseri }\end{array}$ & 0.022 & $<0.001$ & ND & 0.019 & $<0.10$ & ND \\
\hline $\begin{array}{l}\text { ii) } \\
\text { Rekongpeo }\end{array}$ & 0.006 & 0.006 & ND & 0.319 & 0.004 & ND \\
\hline iii) Bajaura & $<0.002$ & 0.005 & ND & 0.006 & 0.010 & ND \\
\hline $\begin{array}{c}\text { iv) } \\
\text { Mashobra }\end{array}$ & 0.009 & 0.004 & ND & 0.037 & 0.005 & $0.011^{*}$ \\
\hline Range & $\begin{array}{c}(<0.002- \\
0.022)\end{array}$ & $\begin{array}{c}(<0.001- \\
0.006)\end{array}$ & ND & $\begin{array}{l}(0.006- \\
0.319)\end{array}$ & $\begin{array}{l}\text { (BDL - } \\
0.010)\end{array}$ & ND \\
\hline \multicolumn{7}{|l|}{ Mango } \\
\hline v) Bilaspur & 0.057 & 0.034 & 0.021 & 0.073 & $<0.10$ & ND \\
\hline \multicolumn{7}{|l|}{ Plum } \\
\hline vi) Solan & $0.003 * * *$ & 0.022 & ND & $<0.002$ & 0.004 & ND \\
\hline \multicolumn{7}{|l|}{ Kinnow } \\
\hline vii) Jachh & $0.003^{* *}$ & $<0.001$ & ND & 0.006 & $<0.10$ & ND \\
\hline $\begin{array}{c}\text { viii) } \\
\text { Dhaulakuan }\end{array}$ & $<0.002$ & $<0.001$ & ND & $<0.002$ & 0.005 & ND \\
\hline
\end{tabular}

* Fenvalerate

** $\beta$-HCH BDL: Below detectable limit

$* * * \gamma-\mathrm{HCH}$ 
Table.6 Pesticide residues in uncultivated field soils of Himachal Pradesh

\begin{tabular}{|c|c|c|c|c|c|c|c|c|c|c|c|c|c|c|c|c|c|c|c|}
\hline \multirow{2}{*}{$\begin{array}{l}\text { Locati } \\
\text { on }\end{array}$} & \multicolumn{19}{|c|}{ Residues (mg//kg) } \\
\hline & $\begin{array}{l}\alpha- \\
H \\
C \\
H\end{array}$ & $\begin{array}{l}\mathrm{B}- \\
\mathrm{H} \\
\mathrm{C} \\
\mathrm{H}\end{array}$ & $\begin{array}{l}\gamma- \\
\mathrm{H} \\
\mathrm{C} \\
\mathrm{H}\end{array}$ & $\begin{array}{l}\delta- \\
\mathrm{H} \\
\mathrm{C} \\
\mathrm{H}\end{array}$ & $\begin{array}{l}\text { dic } \\
\text { ofal }\end{array}$ & $\begin{array}{c}\mathrm{o}, \mathrm{p} \\
,- \\
\mathrm{D} \\
\mathrm{D} \\
\mathrm{E}\end{array}$ & $\begin{array}{c}\alpha \\
\text { endos } \\
\text { ulfan }\end{array}$ & $\begin{array}{l}\text { p, } \\
\text { p'- } \\
\text { D } \\
\text { D } \\
\text { E }\end{array}$ & $\begin{array}{c}\text { B } \\
\text { endos } \\
\text { ulfan }\end{array}$ & $\begin{array}{c}\text { p,p } \\
\text { - } \\
\text { D } \\
\text { D } \\
\text { D }\end{array}$ & $\begin{array}{c}\mathrm{o}, \mathrm{p} \\
,- \\
\mathrm{D} \\
\mathrm{D} \\
\mathrm{T}\end{array}$ & $\begin{array}{l}\text { endos } \\
\text { ufan } \\
\text { sulph } \\
\text { ate }\end{array}$ & $\begin{array}{c}\mathrm{p}, \mathrm{p} \\
,- \\
\mathrm{D} \\
\mathrm{D} \\
\mathrm{T}\end{array}$ & $\begin{array}{l}\text { chlorp } \\
\text { yrifos- } \\
\text { methyl }\end{array}$ & $\begin{array}{l}\text { chlorp } \\
\text { yrifos }\end{array}$ & $\begin{array}{c}\mathrm{o}, \mathrm{p} \\
- \\
\mathrm{D} \\
\mathrm{D} \\
\mathrm{D}\end{array}$ & $\begin{array}{l}\text { cyp } \\
\text { er } \\
\text { met } \\
\text { hrin }\end{array}$ & $\begin{array}{c}\alpha \\
\text { Mert } \\
\text { hrin }\end{array}$ & $\begin{array}{c}\text { B- } \\
\text { Cyflu } \\
\text { thrin }\end{array}$ \\
\hline $\begin{array}{l}\text { Kuku } \\
\text { mseri }\end{array}$ & $\begin{array}{c}0.0 \\
02\end{array}$ & $\begin{array}{c}0.0 \\
09\end{array}$ & $\begin{array}{c}0.0 \\
03\end{array}$ & $\begin{array}{l}\mathrm{N} \\
\mathrm{D}\end{array}$ & $\begin{array}{c}0.0 \\
25\end{array}$ & $\begin{array}{l}\mathrm{N} \\
\mathrm{D}\end{array}$ & 0.005 & $\begin{array}{c}0.0 \\
03\end{array}$ & ND & $\begin{array}{l}\mathrm{N} \\
\mathrm{D}\end{array}$ & $\begin{array}{l}\mathrm{N} \\
\mathrm{D}\end{array}$ & ND & $\begin{array}{l}\mathrm{N} \\
\mathrm{D}\end{array}$ & ND & ND & $\begin{array}{l}\mathrm{N} \\
\mathrm{D}\end{array}$ & ND & ND & ND \\
\hline $\begin{array}{l}\text { Recko } \\
\text { ng Peo }\end{array}$ & $\begin{array}{l}\mathrm{N} \\
\mathrm{D}\end{array}$ & $\begin{array}{l}\mathrm{N} \\
\mathrm{D}\end{array}$ & $\begin{array}{l}0.0 \\
02\end{array}$ & $\begin{array}{l}0.0 \\
02\end{array}$ & $\begin{array}{c}0.0 \\
06\end{array}$ & $\begin{array}{l}0.0 \\
04\end{array}$ & 0.004 & $\begin{array}{c}0.0 \\
02\end{array}$ & 0.016 & $\begin{array}{l}\mathrm{N} \\
\mathrm{D}\end{array}$ & $\begin{array}{l}\mathrm{N} \\
\mathrm{D}\end{array}$ & 0.029 & $\begin{array}{l}\mathrm{N} \\
\mathrm{D}\end{array}$ & ND & ND & $\begin{array}{l}\mathrm{N} \\
\mathrm{D}\end{array}$ & ND & ND & ND \\
\hline $\begin{array}{l}\text { Bajaur } \\
\text { a }\end{array}$ & $\begin{array}{l}\mathrm{N} \\
\mathrm{D}\end{array}$ & $\begin{array}{l}\mathrm{N} \\
\mathrm{D}\end{array}$ & $\begin{array}{l}\mathrm{N} \\
\mathrm{D}\end{array}$ & $\begin{array}{l}\mathrm{N} \\
\mathrm{D}\end{array}$ & $\begin{array}{c}0.0 \\
04\end{array}$ & $\begin{array}{l}\mathrm{N} \\
\mathrm{D}\end{array}$ & 0.003 & $\begin{array}{c}0.0 \\
49\end{array}$ & 0.002 & $\begin{array}{c}0.0 \\
70\end{array}$ & $\begin{array}{c}0.0 \\
15\end{array}$ & ND & $\begin{array}{l}\mathrm{N} \\
\mathrm{D}\end{array}$ & ND & ND & $\begin{array}{l}\mathrm{N} \\
\mathrm{D}\end{array}$ & ND & ND & ND \\
\hline $\begin{array}{l}\text { Masho } \\
\text { bra }\end{array}$ & $\begin{array}{l}\mathrm{N} \\
\mathrm{D}\end{array}$ & $\begin{array}{l}\mathrm{N} \\
\mathrm{D}\end{array}$ & $\begin{array}{l}\mathrm{N} \\
\mathrm{D}\end{array}$ & $\begin{array}{l}\mathrm{N} \\
\mathrm{D}\end{array}$ & ND & $\begin{array}{l}\mathrm{N} \\
\mathrm{D}\end{array}$ & ND & $\begin{array}{l}\mathrm{N} \\
\mathrm{D}\end{array}$ & ND & $\begin{array}{c}0.1 \\
96\end{array}$ & $\begin{array}{l}\mathrm{N} \\
\mathrm{D}\end{array}$ & ND & $\begin{array}{l}\mathrm{N} \\
\mathrm{D}\end{array}$ & ND & ND & $\begin{array}{l}\mathrm{N} \\
\mathrm{D}\end{array}$ & ND & $\begin{array}{c}0.02 \\
6\end{array}$ & ND \\
\hline $\begin{array}{l}\text { Bilasp } \\
\text { ur }\end{array}$ & $\begin{array}{c}0.0 \\
03\end{array}$ & $\begin{array}{l}\mathrm{N} \\
\mathrm{D}\end{array}$ & $\begin{array}{c}0.0 \\
13\end{array}$ & $\begin{array}{l}\mathrm{N} \\
\mathrm{D}\end{array}$ & $\begin{array}{c}0.0 \\
03\end{array}$ & $\begin{array}{l}\mathrm{N} \\
\mathrm{D}\end{array}$ & ND & $\begin{array}{l}0.1 \\
26\end{array}$ & ND & $\begin{array}{l}0.0 \\
35\end{array}$ & $\begin{array}{c}0.0 \\
15\end{array}$ & ND & $\begin{array}{c}0.1 \\
53\end{array}$ & ND & ND & $\begin{array}{l}\mathrm{N} \\
\mathrm{D}\end{array}$ & ND & ND & ND \\
\hline Solan & $\begin{array}{c}0.0 \\
08\end{array}$ & $\begin{array}{l}\mathrm{N} \\
\mathrm{D}\end{array}$ & $\begin{array}{c}0.0 \\
04\end{array}$ & $\begin{array}{l}\mathrm{N} \\
\mathrm{D}\end{array}$ & ND & $\begin{array}{l}\mathrm{N} \\
\mathrm{D}\end{array}$ & ND & $\begin{array}{c}0.0 \\
05\end{array}$ & 0.003 & $\begin{array}{l}\mathrm{N} \\
\mathrm{D}\end{array}$ & $\begin{array}{l}\mathrm{N} \\
\mathrm{D}\end{array}$ & ND & $\begin{array}{c}0.0 \\
06\end{array}$ & 0.005 & ND & $\begin{array}{c}0.0 \\
08\end{array}$ & $\begin{array}{c}0.09 \\
7\end{array}$ & $\begin{array}{c}0.00 \\
9\end{array}$ & 0.034 \\
\hline $\begin{array}{l}\text { Dhaul } \\
\text { akuan }\end{array}$ & $\begin{array}{l}\mathrm{N} \\
\mathrm{D}\end{array}$ & $\begin{array}{l}\mathrm{N} \\
\mathrm{D}\end{array}$ & $\begin{array}{c}0.0 \\
03\end{array}$ & $\begin{array}{l}\mathrm{N} \\
\mathrm{D}\end{array}$ & ND & $\begin{array}{l}\mathrm{N} \\
\mathrm{D}\end{array}$ & 0.006 & $\begin{array}{c}0.0 \\
05\end{array}$ & ND & $\begin{array}{l}\mathrm{N} \\
\mathrm{D}\end{array}$ & $\begin{array}{l}\mathrm{N} \\
\mathrm{D}\end{array}$ & ND & $\begin{array}{l}\mathrm{N} \\
\mathrm{D}\end{array}$ & ND & ND & $\begin{array}{l}\mathrm{N} \\
\mathrm{D}\end{array}$ & ND & ND & ND \\
\hline Jachh & $\begin{array}{l}\mathrm{N} \\
\mathrm{D}\end{array}$ & $\begin{array}{l}\mathrm{N} \\
\mathrm{D}\end{array}$ & $\begin{array}{l}\mathrm{N} \\
\mathrm{D}\end{array}$ & $\begin{array}{c}0.0 \\
01\end{array}$ & ND & $\begin{array}{l}\mathrm{N} \\
\mathrm{D}\end{array}$ & ND & NS & ND & $\begin{array}{c}0.0 \\
10\end{array}$ & $\begin{array}{c}0.0 \\
03\end{array}$ & ND & $\begin{array}{l}\mathrm{N} \\
\mathrm{D}\end{array}$ & ND & 0.004 & $\begin{array}{l}\mathrm{N} \\
\mathrm{D}\end{array}$ & ND & ND & ND \\
\hline
\end{tabular}


Table.7 Average pesticide residues in uncultivated soils

\begin{tabular}{|l|c|c|c|c|c|c|}
\hline Crop & $\sum \mathrm{HCH}$ & $\sum$ DDT & Dicofol & $\sum$ Endosulfan & $\begin{array}{c}\sum \text { Chlorpyrifo } \\
\text { s }\end{array}$ & $\sum$ Pyrethoids \\
\hline Kukumseri & 0.014 & 0.003 & 0.025 & 0.005 & $<0.010$ & $<0.010$ \\
\hline Recongpeo & 0.004 & 0.006 & 0.006 & 0.049 & $<0.010$ & $<0.010$ \\
\hline Bajaura & $<0.002$ & 0.134 & 0.004 & 0.005 & $<0.010$ & $<0.010$ \\
\hline Mashobra & $<0.002$ & 0.196 & $<0.004$ & $<0.002$ & $<0.010$ & $0.026^{* *}$ \\
\hline Bilaspur & 0.016 & 0.329 & 0.003 & $<0.002$ & $<0.010$ & $<0.010$ \\
\hline Solan & 0.012 & 0.019 & $<0.004$ & 0.003 & 0.005 & $0.140^{*}$ \\
\hline & 0.003 & 0.005 & $<0.004$ & 0.006 & $<0.010$ & $<0.010$ \\
\hline $\begin{array}{l}\text { Dhaulakua } \\
\text { n }\end{array}$ & & & & & & \\
\hline Jachh & 0.001 & 0.013 & $<0.004$ & $<0.002$ & 0.004 & $<0.010$ \\
\hline Range & $(<0.002-$ & $(0.003-$ & $(<0.004-$ & $(<0.002-$ & $(<0.010-$ & $(<0.010-$ \\
\hline
\end{tabular}

BDL= Below detectable limit; $* 0.097$ cypermethrin +0.009 alpha-methrin +0.034 cyfluthrin; $* * \alpha$ - Methrin 
The soil samples from Solan were found contaminated with $\alpha-\mathrm{HCH}(0.008 \mathrm{mg} / \mathrm{kg}), \gamma-$ $\mathrm{HCH} \quad(0.004 \mathrm{mg} / \mathrm{kg}), \quad \mathrm{p}, \mathrm{p}$ '-DDE $\quad(0.005$ $\mathrm{mg} / \mathrm{kg}), \quad \beta$-endosulfan $(0.003 \mathrm{mg} / \mathrm{kg}), \mathrm{p}, \mathrm{p}$ DDT $(0.006 \mathrm{mg} / \mathrm{kg})$, chlorpyrifos-methyl (0.005 mg/kg), p,p'-DDD (0.008 mg/kg), cypermethrin $\quad(0.097 \mathrm{mg} / \mathrm{kg}), \quad \alpha$-methrin $(0.009 \mathrm{mg} / \mathrm{kg})$ and $\beta$-cyfluthrin $(0.034$ $\mathrm{mg} / \mathrm{kg}$ ).

The Dhaulakuan soils were observed to contain the residues of $\gamma-\mathrm{HCH}(0.003 \mathrm{mg} / \mathrm{kg})$, $\alpha$-endosulfan $(0.006 \mathrm{mg} / \mathrm{kg})$ and $\mathrm{p}, \mathrm{p}$ '-DDE $(0.005 \mathrm{mg} / \mathrm{kg})$. However, in the soils of Jachh the residues of $\delta$-HCH, p,p'-DDD, o,p'-DDT and chlorpyrifos were detected as 0.001, $0.010,0.003$ and $0.004 \mathrm{mg} / \mathrm{kg}$, respectively.

The data presented in Table 7 show that all samples collected from different locations of Himachal Pradesh contained residues of $\mathrm{HCH}$, DDT, endosulfan and dicofol. The concentrations of $\Sigma$-DDT residues varied from $0.003-0.329 ; \Sigma-\mathrm{HCH}$, from $<0.002$ to $0.016 ; \sum$-endosulfan $<0.002$ to 0.049 and dicofol $(<0.004$ to $0.025 \mathrm{mg} / \mathrm{kg})$.

Among pyrethroids, cypermethrin (0.097 $\mathrm{mg} / \mathrm{kg}), \beta$-cyfluthrin $(0.034 \mathrm{mg} / \mathrm{kg})$ and $\alpha$ methrin $(0.009 \mathrm{mg} / \mathrm{kg})$ were detected in Solan soils while Mashobra soil was found to be contaminated with $\alpha$-methrin $(0.026 \mathrm{mg} / \mathrm{kg})$.

In conclusion, a major fraction of any agricultural pesticide, no matter how applied, eventually finds its way to the soil and it is in the soil that much of the ultimate decomposition takes place. In the present study, more than 90 per cent soil samples analysed from different zones of the state have shown presence of various pesticide residues viz., DDT, $\mathrm{HCH}$, endosulfan, chlorpyrifas, pyrethroids dicofal and chlorothalonil. The frequency of their occurrence was recorded in the order; DDT $25.35 \%>\mathrm{HCH} 21.12 \%$ >endosulfan $20.42 \%$
$>$ chlorpyrifos $14.78 \%>$ dicofol $10.56 \%>$ pyrethroids $6.33 \%$ and chlorothalonil $1.40 \%$. Among $\mathrm{HCH}$ isomers, $\gamma-\mathrm{HCH}$ was detected in all the locations followed by $\alpha-\mathrm{HCH}, \delta-\mathrm{HCH}$ and $\beta-\mathrm{HCH}$, which were found in 7, 6 and 4 locations, respectively. In case of DDT, the metabolite $\mathrm{p}, \mathrm{p}$ '-DDE was encountered more frequently in all the locations while $\mathrm{p}, \mathrm{p}$ '-DDT (parent compound) was detected in 6 locations. Among organophosphorus insecticides only chlorpyrifos could be detected in seven locations except Bilaspur. The contamination of synthetic pyrethroids has also been found at low level. Among these $\alpha$-methrin was the major contaminant followed by cypermethrin, $\beta$-cyfluthrin and fenvalerate. The only fungicide chlorothalonil 0.004 and $0.058 \mathrm{mg} / \mathrm{kg}$ was detected in Solan and Jachh soils, respectively. Apple orchard soils were found to contain highest ensdosulfan residues followed by $\mathrm{HCH}$ and DDT residues. In mango orchard soils dicofol was detected in addition to the apple soil contaminants.

\section{References}

Agnihotri, N. P. 1999. Pesticide safety evaluation and monitoring. New Delhi, IARI, $15 \mathrm{p}$.

Bakshi, Deepali. 2016. Assessment of pesticide residues in integrated farming systems of dry temperate zone. M.Sc. Thesis, Dr. Yashwant Singh Parmar University of Horticulture and Forestry Solan (Nauni) HP. pp. 73.

Banshtu, Tanuja., Patyal, S. K., and Chandel, R. S. 2015. Persistence of profenofos and cypermethrin in tomato grown under mid hill conditions of Himachal Pradesh. The Ecoscan, 9(3\&4): 755759.

Barriada-Pereira, Mercedes., Gonzalez-Castro Maria, J., Muniategui-Lorenzo, Soledad., Lopez-Mahia, Purification., Prada-Rodríguez, Dario., and 
Fernandez-Fernandez, Esther. 2005. Organochlorine pesticides accumulation and degradation products in vegetation samples of a contaminated area in Galicia (NW Spain). Chemosphere, 58:1571-1578.

Bishnu Saha, T., Mazumdar, D., Chakrabarti, K., and Chakraborty, A. 2008. Assessment of the impact of pesticide residues on microbiological and biochemical parameters of tea garden soils in India. Journal of Environmental Science and Health Part B, 43: 723731.

Brar, S. P. S., and Sharma, I. D. 2016. Pesticide Residues in Soils of different Agro-climatic Zones under Various Land Usage in Himachal Pradesh. Journal of Tree Sciences, 35(2):23-31. DOI: 10.5958/2455-7129.2016.00005.4.

Concha-Grana, E., Turnes-Carou, M. I., Muniategui-Lorenzo, S., Lopez-Mahia, P., Prada-Rodriguez, D., FernandezFernandez, E. 2006 Evaluation of $\mathrm{HCH}$ isomers and metabolites in soils, leachates, river water and sediments of a highly contaminated area. Chemosphere, 64(4): 588-595.

Dem, S. B., Cobb, J. M., and Mullins, D. E. 2007. Pesticide residues in soil and water from four cotton growing areas of Mali, West Africa. Journal of Agricultural, Food and Environmental Sciences, 1: 1-12.

Dubey, J. K., Kumar, N., Patyal, S. K., and Nath, A. 1999. Monitoring of dithiocarbamate residues as methyl xanthate in apple and tomato. Pesti. Res. J., 11(2): 225-228.

Dubey, J. K., and Stan, H. J. 1998. Second derivative UV-spectroscopic determination of dithiocarbamate residues as methyl xanthate in apple and lamb's lattuce. J. Food Sci. Technol., 35(6): 482-485

Frank, R., Braun, H. E., Ishida, K., and Suda,
P. 1976. Persistent organic and inorganic pesticide residues in orchard soils and vineyard of south Ontario. Can. J. Soil Sci., 56: 463-484

Gong, Z. M. I., Tao, S., Xu, F. L., Dawson, R., Liu, W. X., Cui, Y. H., Cao, J., Wang, X. J., Shen, W. R., Zhang, W. J., Qing, B. P., and Sun, R. 2004 Level and distribution of DDT in surface soils from Tianjing, China. Chemosphere, 54: 1247-1253.

Harris, C. R., and Sans, W. W. 1969. Vertical distribution of residues of organochlorine insecticides in soils collected from 6 farm in south western Ontario. Proc. Entomol. Soc., 100: 156164

Harris, C. R. and Sans, W. W. 1971. Insecticides residues in soil on 16 farm in western Ontario - 1964, 1966 and 1969. Pestic. Monit. J., 5: 259-267

Jain. H. K., and Agnihotri, N. P. 1986. The persistence of insecticides in soil. Proc. Sym. Pest. Resid. \& Env. Pollu. pp. 114.

Jayashree, R., and Vasudevan, N. 2006. Residues of organochlorine pesticides in agricultural soils of Thiruvallur district, India. Journal of Food, Agriculture and Environment. 4(1): 313-316.

Kannan, K., Battula, S., Loganathan, B. G., Hong, C. S., Li, W. H., Villeneuve, D. L. 2003. Trace organics contaminants, including toxaphene and trifluralin, in cotton field soils from Georgia and South Carolina, USA. Archives of Environmental Contamination and Technology, 45: 30-36.

Kumari, B., Madan, V. K., Singh, J., Singh, S., and Kathpal, T. S. 2004. Monitoring of pesticidal contamination of farmgate vegetables from Hisar. Environmental Monitoring and Assessment, 90: 65-71.

Manirakiza, P., Akinbamijo, O., Covaci, A., Pitonzo, R., and Schepens, P. 2003. Assessment of organochlorine pesticide 
residues in West African city farms: Banjul and Dakar case study. Archives of Environmental Conntamination and Toxicology, 44: 171-179.

Murugan, A. V., Swarnam, T. P. and Gnanasambandan, S. 2013. Status and effect of pesticide residues in soils under different land uses of Andaman islands, India. Environmental Monitoring and Assessment, 185: 81358145.

Sharma, I. D., Chandel, R. S., and Brar, S. S.
2015. Pesticide residues assessment in soils and water from apple growing regions of Himachal Pradesh, India. Journal of Insect Science, 28(1): 126131.

Wang, X., Wang, D., Qin, X., and Xu, X. 2007. Residues of organochlorine pesticides in surface soils from college school yards in Beijing, China Journal of Environmental Sciences, 20:10901096.

\section{How to cite this article:}

Sukh Pal Singh Brar and Sharma, I.D. 2019. Soil Pesticide Residues in Orchard based Land Use Systems across Different Agro-climatic Zones of Himachal Pradesh. Int.J.Curr.Microbiol.App.Sci. 8(04): 1250-1263. doi: https://doi.org/10.20546/ijcmas.2019.804.144 